\title{
Novel Approach for Ensuring Increased Validity in Home Blood Pressure Monitoring
}

\author{
Stefan Wagner \\ Aarhus School of Engineering \\ Aarhus University \\ Dalgas Avenue 2, Aarhus, Denmark \\ sw@iha.dk
}

\author{
Thomas Skjødeberg Toftegaard \\ Aarhus School of Engineering \\ Aarhus University \\ Aabogade 34, Aarhus, Denmark \\ tst@cs.au.dk
}

\author{
Olav W. Bertelsen \\ Department of Computer Science \\ Aarhus University \\ Aabogade 34, Aarhus, Denmark \\ olavb@cs.au.dk
}

\begin{abstract}
- this paper proposes a novel technique to increase the validity of home blood pressure monitoring by using various sensor technologies as part of an intelligent environment platform in the home of the user. A range of recommendations exists on how to obtain a valid blood pressure but with the devices currently available it cannot be verified whether a user is actually following the recommendations or not. An initial prototype is presented implementing part of the proposed solution including a limited pilot study as a status on the work in progress. Results indicate that the solution is technical feasible.
\end{abstract}

Keywords- pervasive healthcare; telemedicine; hypertension; home blood pressure; recommendations; white coat effect;

\section{INTRODUCTION}

Blood pressure is a principal vital sign and the measurement hereof is one of the most basic medical assessments [1]. Blood pressure is the pressure exerted by circulating blood on the walls of the blood vessels. Elevated blood pressure above $140 / 90 \mathrm{mmHg}$ is called hypertension, which can be a chronic medical condition. Persistent hypertension is one of the risk factors for strokes, heart attacks, heart and kidney failure and other heart and circulatory diseases [2]. As there are usually no symptoms, measurements of the blood pressure are highly relevant for high-risk groups. The level of the blood pressure is the main factor in the decision to start antihypertensive therapy. It is thus vital that the measurements are obtained in a valid manner.

Measurements can be performed at the clinic, as well as in the home. In the latter case, this may be done by using either ambulatory blood pressure devices which must be worn by the user 24-hours a day, or automatic home blood pressure devices, either wrist- or bicep-fixated, for regular or casual readings. Both ambulatory and home devices have proven successful for obtaining valid measurements in clinical trials [1]. Duplicate home blood pressure measurements taken in the morning and evening for one week and recorded and averaged can be utilized for diagnostic and therapeutic purposes [5].

Measurements at the clinic are believed to cause the whitecoat effect in some cases: patients showing higher blood pressure readings at the clinic than at home, which affects as much as $20 \%$ of all patients [4]. Clinical based measurements also requires the user to attend the clinic, which might not be feasible for the chronically ill or disabled, or simply too expensive or impractical for frequent measurements. While ambulatory devices often appear as large and impractical devices, as users need to carry them on their body constantly during the observation period, wrist- and bicep-fixated home measurement devices appear less cumbersome to the user [1].

To obtain valid blood pressure measurements, a range of recommendations exists with regard to how the measurements should be performed. These include having bare-arm contact, sitting down, at least 5 minutes rest prior to the measurement, quiet surroundings (no noise), preferably an empty bladder, and restraining from physical activities, such as talking, working, watching TV and other activities of daily living $[1,5,6]$. As reported by $[5,7]$, the accuracy of patients obtaining and writing down the blood pressure readings has been found to be questionable when unassisted. Devices with printer capabilities or memory are available today.

Errors in measuring blood pressure correctly are reported to occur often. Few studies have reported on the incidence of such faulty measurements in the home and whether the healthcare personnel in general are concerned with the validity of the measurements obtained. Some of the errors result in overestimates of the blood pressure which could cause almost twice as many patients receiving the diagnosis of hypertension [1]. An example of this might be a user not having rested sufficiently before taking the measurement causing an increase in the measured blood pressure of up to $10-15 \mathrm{mmHg}$ systolic pressure, shifting the patient from the category of prehypertension to stage 1 hypertension (e.g. from 135 to 145). In other cases the opposite might occur; hypertension is underdiagnosed owing to underestimates of blood pressure. Many errors result in variability of readings and cause confusion regarding blood pressure status. Despite the importance of blood pressure assessments, current guidelines for patient preparation and measurement technique, also known as the "recommendations", are rarely followed, and the equipment used is often inaccurate [1].

A substantial amount of work has been done on investigating the use of pervasive healthcare technology and telemedicine for guiding users through the measurement process, obtaining the blood pressure data through wired and wireless automatic blood pressure devices, and reporting these data to the stakeholders through standard communication media such as email or web-pages $[10,11,12,13]$. The cited 
studies do not address the uncertainty of whether the home users have actually followed the recommendations for obtaining a valid blood pressure or not, but rely on the users training, or on asking standard questions during measurement. It has not been verified that this approach produces more valid blood pressure readings.

This paper proposes a novel technique to increase the validity of the measurements by using various sensor technologies as part of an intelligent environment platform in the home of the user, the OpenCare Home Intelligent Environment Platform (OPCHIEP), to better guide the user through the recommended process. If we are able to measure different aspects of user activity and context through the various sensors, we might be able to guide the user to e.g. refrain from speaking on the phone or moving around during measurements and ensure other elements of the recommendations for obtaining valid blood pressure are followed. Furthermore, the data obtained may be augmented with meta-data, describing the quality of the measurement, e.g. that the user did indeed rest for 5 minutes before taking the measurement. Amongst relevant factors to measure and include in the meta-data are those concerning the recommendations for obtaining a valid blood pressure, as discussed in the following section. These meta-data may be used to increase the physician's confidence in home-based measurements, and empower him to better evaluate the validity of a patients data, obtained from home monitoring of the blood pressure.

\section{PRELIMINARIES}

\section{A. Recommendations for Home Blood Pressure Monitoring}

Home blood pressure monitoring is claimed to increase in validity if the user observes a range of best-practices before and during the actual blood pressure measurement $[1,3,5,6,8,9]$. A list of 13 steps (TABLE I) has been compiled from these and other sources $[3,6,8,9]$ as a best-practice recommendation

TABLE I. LIST OF RECOMMENDATIONS

\begin{tabular}{|c|l|}
\hline No. & \multicolumn{1}{|c|}{ Recommendation } \\
\hline 1 & $\begin{array}{l}\text { Do not drink coffee or smoke cigarettes 30 minutes before } \\
\text { having your blood pressure measured }\end{array}$ \\
\hline 2 & $\begin{array}{l}\text { Go to the bathroom prior to the reading. A full bladder can } \\
\text { change a blood pressure reading. }\end{array}$ \\
\hline 3 & $\begin{array}{l}\text { Before the test, sit and rest for five minutes with back supported } \\
\text { and your feet flat on the ground. }\end{array}$ \\
\hline 4 & $\begin{array}{l}\text { Make sure of being in quit surroundings and not talking while } \\
\text { doing the reading }\end{array}$ \\
\hline 5 & Rest your arm on a table at the level of your heart \\
\hline 6 & Wear short sleeves so the arm is exposed \\
\hline 7 & $\begin{array}{l}\text { Get two readings, taken at least two minutes apart, and average } \\
\text { the results }\end{array}$ \\
\hline 8 & Record the reading. \\
\hline 9 & Make sure to keep accurate record \\
\hline 10 & Do not round the numbers \\
\hline 11 & Record any medications taken and when \\
\hline 12 & Record whether you have just exercised \\
\hline 13 & Record whether you have just eaten \\
\hline
\end{tabular}

Table I: List of recommendations for obtaining valid home blood pressure measurement. Points 3 to 10 are relevant during the measurement. 1 to 2 are important prerequisites before the measurement, while

This work is supported by a grant from the Danish Ministry of Education and the Engineering College of Aarhus, Aarhus, Denmark list that will be used in this study. For the remainder of this study, we will refer to this list as the "recommended behavior", or the "recommendations", in the context of obtaining a valid blood pressure in the home of the users.

It is important to note, that different organizations, e.g., the American Hypertension Association and British Hypertension Society, have different sounding recommendations, and not all the sources $[3,6,8,9]$ agrees on the same recommendations.

\section{B. Home Blood Pressure Equipment Current Situation}

Today, home blood pressure users, excluding those using ambulatory blood pressure monitoring, typically use one of the recommended home blood pressure devices available. None of these devices are able to ensure that the users are observing all 13 steps of the recommendation, but some have partial support. Typically, they are capable of automatically storing date and time of the measurement, satisfying recommendation nr. 9. Some are able to ensure that the cuff is inflated sufficiently, while others have the ability to average several readings, satisfying recommendation $\mathrm{nr} .8$.

\section{Ensuring recommendations are followed}

In order to meet more of the requirements, we must utilize sensor technology to obtain more information from the context of the user. For instance, we may use an activity sensor for assuring that the user has rested before taking the blood pressure, and a chair occupancy sensor verifying that the user is sitting down, meeting recommendation nr. 3 . We may use a bathroom occupancy sensor for verifying whether the user has gone to the bathroom prior to the reading, verifying recommendation $\mathrm{nr}$. 2. We may use a noise level sensor, for ensuring that the user is in quite surroundings, and is not watching television, engaged in conversation, or other problematic steps, as related to recommendation nr. 4. Other sensor types may be used to obtain additional data from the user context. These will not be discussed in this paper.

\section{SUGGESTED SOLUTION}

We suggest creating a platform for monitoring the activities of the user and to assist the user to obtain a more valid blood pressure measurement. Data will be obtained through automatic home blood pressure monitoring devices via Bluetooth, stored on a base-station in the user's home, and sent to a server for data collection and distribution purposes.

Data received from automatic blood pressure monitoring devices includes: systolic blood pressure, diastolic blood pressure and heart rate. Also, the date and time of the measurement will be attached, as well as device identity.

Other sensors needed include an activity sensor. One option considered is using an accelerometer based sensor with a radio for wireless communication. Here, we suggest using the Intel Shimmer platform running the SPINE framework on the basestation. This platform is already being used in the OPCHIEP framework as a fall detection device. By adding another set of algorithms we may use it as both an activity sensor, as well as a fall detector. 
The meta-data to add to the regular data might include:

1. Validity-index-level

2. Confidence-index-level

3. Context-data

The validity-index-level describes the overall calculated validity of the measurement as well as a number of details (see TABLE II. ). The confidence-index-level gives an indication of the number of supported recommendations on the platform. Are we ensuring compliance for only one type of recommendation or for several? If for instance we are only able to measure the whether the person is seated as well as how much the person is moving during the measurement process, then we have only medium confidence in the reading. If we are not able to say anything at all, we have low confidence. Only if we know all of the recommendations have been complied with can we have high confidence. Only some of the recommendations may be validated through the use of sensors. For those recommendations that may be not verified we may use context-data for manual inspection by the physician wanting to use the data, in case of uncertainty. This might include taking a picture or video-sequence of the user during measurement, for manual inspection. This may be used by the physician if he suspects that the user have not complied with the recommendations.

TABLE II. VALIDITY-INDEX-LEVEL

\begin{tabular}{|c|l|}
\hline No & \multicolumn{1}{|c|}{ Detail of the validity-index-level } \\
\hline 1 & Overall-validity \\
\hline 2 & Coffee-abstained-thirty-minutes \\
\hline 3 & Smoking-abstained-thirty-minutes \\
\hline 4 & Empty-bladder \\
\hline 5 & Five-minutes-rested \\
\hline 6 & Seated-during-measurement \\
\hline 7 & No-sound \\
\hline 8 & Arm-at-rested-position \\
\hline 9 & Arm-at-heart-level \\
\hline 10 & Two-readings-average-obtained \\
\hline
\end{tabular}

Table II: List of the details of the validity-index-level as a measure of the validity of home blood pressure measurement.

\section{METHODS AND MATERIALS}

\section{A. Planned studies}

The suggested solution in section III requires several planned pilot studies in the laboratory for testing and verifying conceptual and technical design concepts before user testing and clinical trials can commence,. The pilot study reported on in this paper aims at testing the technical feasibility of a limited sensor support array and wireless data collection from the sensors, including from the blood pressure device, the activity device and the chair occupancy sensor.

This pilot study is done in the laboratory only, verifying that movement data and blood pressure data can be measured correctly, wirelessly transferred and stored on the base-station for further analysis, and that the pressure mat sensor can be used as a chair occupancy sensor for obtaining whether a user is occupying the chair during measurement or not. The study is restricted to qualitative laboratory tests and does not include end-users of the target group.

\section{B. Materials needed for initial piloty study}

The system is based on elements from the OPCHIEP framework for creating an intelligent environment for impaired users and their helpers, and consists of a base-station with various internal and external sensors attached for creating a context-aware environment.

The base-station is a touch screen computer with network and Bluetooth communication capabilities running a test application written for this pilot study specifically, as well as support drivers and services. The Asus Eee touch PC is using an Intel Atom processor, running Windows XP, using both Java and .NET runtimes. Java for SPINE support and .NET used for Bluetooth blood pressure device and chair occupancy sensor integration (see TABLE III. and TABLE IV. for details).

The base-station platform is also used for data collection and distribution purposes. It communicates with the Omron 750IT BT home blood pressure device using Bluetooth. The movement sensors are based on the Intel Shimmer sensor nodes, and the SPINE framework. This framework provides wireless access for monitoring user activity levels, suitable to determine an overall-activity index.

\section{Piloty sudy preparation, execution and expected results}

Preparation: the pressure mat sensor is placed on a chair. A test-user is equipped with the activity sensor, placed in a pocket. The blood pressure device is placed on a table adjacent to the chair. The touch screen computer is preloaded with the test application for the pilot study, including drivers for the blood pressure device, pressure mat chair sensor and the SPINE activity service application. In phase 1 the user is instructed to walk around for 1 minute, then in phase 2 sit down wait for 1 minute and finally take the blood pressure measurement. This is repeated 10 times.

Execution: user is asked to execute the prepared procedure, while being observed by the facilitator and data is collected by the test application and stored for future reference.

Expected results: it is expected that the activity sensor will report "movement detected" to the test application during phase 1 , then, "no movement detected" during phase 2, where also the chair occupancy sensor will report "chair occupied" and finally that the measured blood pressure data is transferred to the test application in phase 3 ..

TABLE III. SOLUTION HARDWARE COMPONENTS

\begin{tabular}{|l|}
\hline \multicolumn{1}{|c|}{ Hardware Components } \\
\hline Asus Eee touch.computer with Bluetooth dongle, ASUS, USA \\
\hline Blood Pressure Device Omron 750IT BT, Corscience, Germany \\
\hline Intel Shimmer sensor node, Shimmer Research, Ireland \\
\hline Intel Shimmer base-station node, Shimmer Research, Ireland \\
\hline PhidgetInterfaceKit 8/8/8, Phidgets Inc., USA \\
\hline Defender 1146763 Standard Pressure Mat, Farnell, UK \\
\hline
\end{tabular}

Table III. A list of hardware components used in the initial prototype 
TABLE IV. SOLUTION SOFTWARE COMPONENTS

\begin{tabular}{|l|}
\hline \multicolumn{1}{|c|}{ Software Components } \\
\hline Windows XP operating system, Microsoft, USA \\
\hline .NET Framework, Microsoft, USA \\
\hline Java Framework, Sun, USA \\
\hline SPINE Framework, Telecom Italia, Italy \\
\hline OPCHIEP framework, Aarhus School of Engineering, Denmark \\
\hline SPINE Activity service application for the OPCHIEP framework \\
\hline Windows test application for pilot study 1 (for bpm and mat support) \\
\hline
\end{tabular}

\section{RESULTS}

The completed experiment resulted in the data presented in TABLE V. which turned out almost as expected. One blood pressure measurement did not get transferred to the PC for unknown reasons during experiment 9 .

TABLE V. PILOTY STUDY RESULTS

\begin{tabular}{|c|l|l|l|}
\hline No & $\begin{array}{c}\text { Phase 1: } \\
\text { walking detected } \\
\text { and chiar not } \\
\text { occupied as } \\
\text { expected? }\end{array}$ & $\begin{array}{c}\text { Phase 2: } \\
\text { sitting still and } \\
\text { chair occupied as } \\
\text { expected? }\end{array}$ & $\begin{array}{c}\text { Phase 3: } \\
\text { blood pressure } \\
\text { readings } \\
\text { transferred to PC } \\
\text { as expected? }\end{array}$ \\
\hline 1 & Yes & Yes & Yes \\
\hline 2 & Yes & Yes & Yes \\
\hline 3 & Yes & Yes & Yes \\
\hline 4 & Yes & Yes & Yes \\
\hline 5 & Yes & Yes & Yes \\
\hline 6 & Yes & Yes & Yes \\
\hline 7 & Yes & Yes & Yes \\
\hline 8 & Yes & Yes & Yes \\
\hline 9 & Yes & Yes & No \\
\hline 10 & Yes & Yes & Yes \\
\hline
\end{tabular}

Table V: List of results from the initial pilot study.

Results indicate that it is possible to combine different sensors to obtain various context data. We have obtained data to support validity-index-level details $1,5,6,7$ and 10 from TABLE II.

\section{DISCUSSION}

The results indicate that it is feasible to develop a solution that can combine different sensors in an intelligent environment. It has not yet been verified that the suggested solution (section III) will actually create more valid blood pressure readings. In order to verify this, more laboratory testing is needed. We need to obtain data from more sensor types and more test subjects both while following the recommendations and while not doing so.

It appears that the Omron device at times does not work as specified (see TABLE V. experiment no. 9), but whether the error rests with the Omron device or the test application has not been determined. Also, the Omron device was slow and overly complex to use. We suggest changing to the A\&D blood pressure measurement device for future experiments.

The use of a wireless activity sensor appears feasible, but will users remember to wear the device, and recharge it? During this pilot study we experienced that the device battery was depleted. Thus we might consider an alternative to the activity sensor that will not rely on battery power.

If further pilot study laboratory experiments indicate that we are in fact able to obtain more valid measurements, we plan to continue with a series of clinical trials in the homes of hypertension patients.

\section{ACKNOWLEDGMENT}

Thank you to Martin Kjeldsen (Aarhus School of Engineering) for working on the SPINE Activity service application and providing assistance with deployment.

\section{REFERENCES}

[1] N. R. C. Campbell and D. W. McKay Accurate blood pressure measurement: Why does it matter? Can. Med. Assoc. J., August 1, 1999; 161(3): 277 - 278.

[2] Maton, Anthea; Jean Hopkins, Charles William McLaughlin, Susan Johnson, Maryanna Quon Warner, David LaHart, Jill D. Wright (1993). Human Biology and Health. Englewood Cliffs, New Jersey, USA Prentice Hall. ISBN 0-13-981176-1. OCLC 32308337.

[3] T.G. Pickering and W.B. White (2008). When and how to use self (home) and ambulatory blood pressure monitoring. Journal of the American Society of Hypertension 2(3) (2008) 119-124.

[4] Krakoff, Nancy T. Artinian and David Goff Thomas G. Pickering, Nancy Houston Miller, Gbenga Ogedegbe, Lawrence R. Call to Action on Use and Reimbursement for Home Blood Pressure Monitoring Hypertension 2008;52;1-9; originally published online May 22, 2008.

[5] Pierdomenico SD, Di Nicola M, Esposito AL, et al. (June 2009) Prognostic Value of Different Indices of Blood Pressure Variability in Hypertensive Patients. American Journal of Hypertension 22 (8): 842-7. doi:10.1038/ajh.2009.103. PMID 19498342.

[6] National Heart Lung and Blood Institute, U.S. Department of Health \& Human Services (November 2009). Tips for Having Your Blood Pressure Taken [Online: http://www.nhlbi.nih.gov/hbp/detect/tips.htm].

[7] Mengden T, Chamontin B, Phong Chau NG, Gamiz JLP. Chanudet X and the participants of the First International Consensus Conference on Self-Blood Pressure Measurement. User procedure for self-measurement of blood pressure. Blood Press Monit 2000;5: 111-12.

[8] British Hypertension Socity (November 2009). Blood Pressure Measurement With Electronic Blood Pressure Monitors [Online: http://www.bhsoc.org/bp_monitors/BLOOD_PRESSURE_1784a.pdf].

[9] Measuring Your Blood Pressure at Home (November, 2009) [Online: http://www.blood-pressure-monitoring.org/measuring-bloodpressure.htm].

[10] Cafazzo, J., Leonard, K., Easty, T.;, Rossos, P., Chan, C., Logan A.(2006) Complex tele-monitoring: facilitating patient self-care of the chronically-illIn: Proceedings of the IASTED International Conference on Telehealth, p 158-63, 2006

[11] Rogers, M.A.M., Buchan, D.A., Small, D., Stewart, C.M., Krenzer, B.E. (2002) Telemedicine improves diagnosis of essential hypertension compared with usual care. In: Journal of Telemedicine and Telecare, $v 8$, n 6, 344-9, 2002.

[12] Villalba, E., Peinado, I., Arredondo, M.T. (2009) Self care system to assess cardiovascular diseases at home. In: Lecture Notes in Computer Science (including subseries Lecture Notes in Artificial Intelligence and Lecture Notes in Bioinformatics), v 5615 LNCS, n PART 2, p 248-257, 2009, Universal Access in Human-Computer Interaction: Intelligent and Ubiquitous Interaction Environments - 5th International Conference UAHCI 2009 - Held as Part of HCI International 2009, Proceedings.

[13] Lin, C., Lee, R., Hsiao, C. (2008) A pervasive health monitoring service system based on ubiquitous network technology. In: International Journal of Medical Informatics, v 77, n 7, p 461-469, July 2008. 